\title{
Queueing systems with different types of renovation mechanism and thresholds as the mathematical models of active queue management mechanism
}

\author{
Hilquias Viana Carvalho Cravid ${ }^{1}$, \\ Ivan S. Zaryadov ${ }^{1,2}$, Tatiana A. Milovanova ${ }^{1}$ \\ ${ }^{1}$ Peoples' Friendship University of Russia (RUDN University) \\ 6, Miklukho-Maklaya St., Moscow, 117198, Russian Federation \\ ${ }^{2}$ Institute of Informatics Problems, FRC CSC RAS \\ 44-2, Vavilova St., Moscow 119333, Russian Federation
}

(received: October 27, 2020; accepted: November 12, 2020)

This article is devoted to some aspects of using the renovation mechanism (different types of renovation are considered, definitions and brief overview are also given) with one or several thresholds as the mathematical models of active queue management mechanisms.

The attention is paid to the queuing systems in which a threshold mechanism with renovation is implemented. This mechanism allows to adjust the number of packets in the system by dropping (resetting) them from the queue depending on the ratio of a certain control parameter with specified thresholds at the moment of the end of service on the device (server) (in contrast to standard RED-like algorithms, when a possible drop of a packet occurs at the time of arrivals of next packets in the system).

The models with one, two and three thresholds with different types of renovation are under consideration. It is worth noting that the thresholds determine not only from which place in the buffer the packets are dropped, but also to which the reset of packets occurs. For some of the models certain analytical and numerical results are obtained (the references are given), some of them are only under investigation, so only the mathematical model and current results may be considered.

Some results of comparing classic RED algorithm with renovation mechanism are presented.

Key words and phrases: random early detection, active queue management, queuing system, general renovation, threshold mechanism, drop function, congestion control

(C) Viana Carvalho Cravid H., Zaryadov I. S., Milovanova T. A., 2020 


\section{Introduction}

In modern communication networks the problem of congestion avoidance does not have a satisfying solution [1] and the development of active queue management (AQM) algorithms appears to be the actual task for researches and practitioners.

A numerous number of AQM schemes have been proposed [2], some of them were investigated and standardised by IETF working group on "Active Queue Management and Packet Scheduling" [3].

For the most AQM algorithms models the performance analysis is performed by simulation (for example, [2]) and that is why the bridges between the available use-case results and the available analytic results are very few (see, for example, [4]-[7]).

In this paper the mathematical models of RED-like ( in contrast to standard RED algorithm, when a possible reset occurs at the time of the next packet arrival and the control parameter is an exponentially weighted average queue length [8]-[10], in our models the decision about a possible packet drop is made at the momemnts of service completions) algorithms with renovation and one or several thresholds (which determine not only the place in the buffer from which the packets are dropped, but also the place to which the reset of packets occurs).

The structure of the article is following. The section 2 gives the brief description active queue algorithms, especially of the classic RED algorithm. The section 3 consists of foyr subsections: in 3.1 the definitions of different types of renovation are given as well as a brief overview of queueing systems with renovation; in 3.2 the models with renovation and a single thresholds are described; the subsectionn 3.3 is devoted to models with renovation and two thresholds (for one of these models some analitycal results are presented); the last subsection 3.3 describes renovation models with three thresholds. In section 4 the comparison of results for RED algorithm and some renovation models is presented, based on experimental results from [11]-[13]. These results confirm assumptions that the use of the renovation mechanism in the single server queues under heavy overload conditions and some other constraints allows one to achieve at least the same performance level as by the classical random early detection algorithm. The last section 5 concludes the paper with the short discussion.

\section{Active queue management algorithms. The brief description of RED algorithm module}

According to RFC 7567 [1] active queue management (AQM) is considered as a best practice of network congestion avoidance (reducing) in Internet routers. The active queue management is the policy of dropping packets inside a buffer associated with a network interface controller (NIC) before that buffer becomes full (or gets close to becoming full) and this policy is based on some rules (algorithms ) such as:

- Random Early Detection (random early discard or random early drop) (RED) [8]-[10], [14] — is a queuing discipline for a network scheduler suited for congestion avoidance by pre-emptively dropping packets before 
the buffer becomes completely full based on predictive models to decide which packets to drop;

- Explicit Congestion Notification (ECN) [9], [15] and its modifications [16][19] - allows end-to-end notification of network congestion without dropping packets (opposed to RED);

- controlled delay (CoDel) [20] and its modifications [21], [22] — a scheduling algorithm for the network scheduler, designed to overcome bufferbloat in networking hardware by setting limits on the delay network packets experience as they pass through buffers in this equipment;

- BLUE [23] and its modifications [24]-[26] — operates by randomly dropping or marking packet with explicit congestion notification mark before the transmit buffer of the network interface controller overflows and it requires little or no tuning to be performed by the network administrator;

- CAKE (Common Applications Kept Enhanced)[27], [28] — is a shapingcapable queue discipline which uses both AQM and FQ, itcombines COBALT, which is an AQM algorithm combining Codel and BLUE

Early AQM disciplines (notably RED and SRED) require careful tuning of their parameters in order to provide good performance, but modern AQM disciplines (Blue, CoDel, CAKE and new modifications of RED - the overview may be seen in [29], [30]) are self-tuning, so they can be run with their default parameters.

The classic RED [8] is an active queue management algorithm with two thresholds $\left(Q_{\min }\right.$ and $\left.Q_{\max }\right)$, which monitors the average queue size and drops packets (or marks packets when it used in conjunction with ECN) based on statistical probabilities $p(\hat{Q})[8]$ :

$$
p(\hat{Q})=\left\{\begin{array}{l}
0, \quad 0 \leqslant \hat{Q} \leqslant Q_{\min }, \\
\frac{\hat{Q}-Q_{\min }}{Q_{\max }-Q_{\min }} p_{\max }, \quad Q_{\min }<\hat{Q} \leqslant Q_{\max }, \\
1, \quad \hat{Q}>Q_{\max },
\end{array}\right.
$$

where $p_{\max }$ is the maximum of dropped probability (probability of marking), and $\hat{Q}$ is the queue length control parameter (low-pass filter) calculated by formula:

$$
\hat{Q}_{k+1}=\left(1-w_{q}\right) \hat{Q}_{k}+w_{q} \hat{Q}_{k}, \quad k=0,1,2, \ldots,
$$

here $w_{q}\left(0<w_{q}<1\right)$ is a weight coefficient of the exponentially weighted moving-average.

\section{Renovation mechanism with thresholds}

\subsection{The definition of renovation mechanism}

The renovation mechanism was introduced in [31]: at the moment of the end of its service the packet on the server may either just leave the system with some non-zero probability $p$, or may empty the buffer with the renovation 
probability $q=1-p$. In [31] the steady-state probability distributions for several types of queueing systems were presented.

Later the model of renovation with feedback (or repeated service) was introduced in 2007 by professor P. P. Bocharov in [32]. The renovation with feedback means that after emptying the buffer with probability $q$ the served packet starts another round of service. The main characteristics in matrixanalytical form were obtained. Another system with renovation and feedback was studied in [33], [34], where the retrial queueing system with renovation and recurrent input flow was investigated.

The generalisation of renovation mechanism was proposed in 2008 by professor A. V. Pechinkin [35]: at the moment of the end of service the packet may drop from the buffer with probability $q(i)$ exactly $i(i \geqslant 1)$ other packets and leaves the system or may just just leave the system without any effect on it with the complementary probability $p=1-\sum_{i=1}^{r} q(i)$.

In [12], [13], [35]-[39] queueing systems with different types of renovation and service disciplines were presented. It was proved that for $G i / M / n / \infty$ systems with general renovation (or just renovation) the steady-state probability distribution has the geometric form and sojourn time distribution of a served (lossed) packet has an exponential form.

The study of queueing systems with renovation mechanism and thresholds was started in [12], [13].

\subsection{The mathematical model with renovation and one threshold}

The simplest mathematical models of renovation mechanism with thresholds are the models with one threshold $Q_{1}$ for queue length.

If the current queue length $\hat{q}$ is larger than $Q_{1}\left(\hat{q}>Q_{1}\right)$ then a packet on the server at the moment of the end of its service may either leave the system with probability $p$ or may drop a packet (packets) from the buffer and leave (renovation mechanism).

The next types of renovation may be considered:

- the "simple" renovation of the first type - the fixed size group of $k$ $(k \geqslant 1)$ packets will be dropped with fixed probability $q_{k}$;

— the "simple" renovation of the second type - the fixed size group of $k$ $(k \geqslant 1)$ packets will be dropped with fixed probability $q_{k}$ (but $Q_{1}$ will remain anyhow)

- the "complete" ("full") renovation (or just renovation) - all packets from the buffer will be dropped with probability $q=1-p$, the system will be empty;

- the "incomplete" renovation - all packets (that are after the threshold) willbe dropped with probability $q=1-p, Q_{1}$ packets will remain in the system;

- the general renovation of the first type - exactly $k(k \geqslant 1)$ packets from the buffer will be dropped with probability $q(k)$ if in the buffer there are more then $k$ packets or the system will be empty if in the buffer were were less (or equal) then $k$ packets;

- the general renovation of the second type - exactly $k(k \geqslant 1)$ packets from the buffer may be dropped with probability $q(k)$ if there were more 
then $k+Q_{1}$ packets in the buffer, or $Q_{1}$ packets will remain in the system if in the buffer were less (or equal) then $k+Q_{1}$ packets.

For the last two cases of general renovation the following condition on probabilies $p$ and $q(k)(k \geqslant 1)$ must be met:

$$
p+\sum_{k=1}^{\infty} q(k)=1,
$$

$p$ is the probability, that a served packet will just leave the system without dropping other packets.

The minus of the first, the third and the fifth drop mechanism is that too many packets may be dropped, the minus of the second, the fourth and the sixth drop mechanism is that the buffer may remain overflowed. Models with two thresholds allow to get rid of these minuses.

\subsection{The mathematical model with renovation and two thresholds}

In this part of the article we will discuss models of RED-like algorithm based on queueing systems with renovation and two thresholds.

The thresholds of the first type: the first threshold $Q_{1}$ determines when packets start to be dropped, the second threshold $Q_{2}\left(Q_{2}>Q_{1}\right)$ sets the maximum value of drop probability.

The types of renovation policy. At the moment of the end of a packet service the current queue length $\hat{q}$ is compared with thresholds and if $\hat{q} \leqslant Q_{1}$ then no one of the packets from the buffer is dropped.

- If $Q_{1}+1 \leqslant \hat{q} \leqslant Q_{2}$ then the fixed size group of $k(k \geqslant 1)$ packets will be dropped with fixed probability $q_{k}$. If $\hat{q} \geqslant Q_{2}+1$ then the fixed size group of $k$ packets in the buffer will be dropped with maximal probability $q_{\max }\left(q_{k}<q_{\max } \leqslant 1\right.$.

- If $Q_{1}+1 \leqslant \hat{q} \leqslant Q_{2}$ then the fixed size group of $k$ packets will be dropped with probability $q_{k}(\hat{q}), 0<q_{k}(\hat{q})<q_{\max }$. If $\hat{q} \geqslant Q_{2}+1$ then the fixed size group of $k$ packets in the buffer will be dropped with maximal probability $q_{\max }$ (this model was considered in [12] for $k=1$ (the last packet is dropped) and the steady-state probability distribution of packets in the system(for imbedded Markov chain) as well as some probabilistic (the probability that the arriving packet will be dropped or served) and sojourn time characteristics for served packets were obtained).

- If $Q_{1}+1 \leqslant \hat{q} \leqslant Q_{2}$ then the fixed size group of $k$ packets will be dropped with fixed probability $q_{k}$. If $\hat{q} \geqslant Q_{2}+1$ then all packets except the first $Q_{1}$ will be dropped with probability $q$.

- If $Q_{1}+1 \leqslant \hat{q} \leqslant Q_{1}$ then the fixed size group of $k$ packets will be dropped with probability $q_{k}(\hat{q})$. If $\hat{q} \geqslant Q_{2}+1$ then all packets except the first $Q_{1}$ will be dropped with probability $q$.

- If $Q_{1}+1 \leqslant \hat{q} \leqslant Q_{2}$ then the arbitrary size group of $k$ packets will be dropped with given probability $q(k)(0<q(k)<1)$. If $\hat{q} \geqslant Q_{2}+1$ then all packets except the first $Q_{1}$ will be dropped with probability $q$. 
For all types of renovation policy the following condition on probabilies $q_{k}$, $q_{k}(\hat{q}), q(k), q_{\max }, q(k \geqslant 1)$ must be met:

$$
p_{k}+q_{k}=1, \quad p_{k}(\hat{q})+q_{k}(\hat{q})=1, \quad k \geqslant 1, \quad p+\sum_{k=1}^{\infty} q(k)=1,
$$

where $p_{k}, p_{k}(\hat{q})(k \geqslant 1)$ and $p$ are the probabilities that the served packet will leave the system without dropping any other packets from the buffer (if $\left.Q_{1}+1 \leqslant \hat{q} \leqslant Q_{2}\right)$, and $p_{\max }^{(2)}+q_{\max }=1, p^{(2)}+q=1, p_{\max }^{(2)}$ and $p^{(2)}$ are the probabilities that the served packet will leave the system without dropping any other packets from the buffer (if $\hat{q} \geqslant Q_{2}+1$ ).

The thresholds of the second type: the first threshold $Q_{1}$ determines when packets start to be dropped, the second threshold $Q_{2}$ determines the place to which packets will be dropped from the queue $\left(Q_{1} \geqslant Q_{2}\right)$. If the current queue size $\hat{q}$ is less than $Q_{1}$, then a served packet just leave the system. But if $\hat{q} \geqslant Q_{1}+1$ then the following types of renovation (dropping) mechanism may be applied:

- the fixed size group of $k(k \geqslant 1)$ packets will be dropped with fixed probability $q_{k}$ (anyhow $Q_{2}$ packets will remain in system);

— with probability $q$ only $Q_{2}$ packets will remain in system, all others packets will be dropped;

- the arbitrary size group of $k$ packets will be dropped with given probability $q(k)(0<q(k)<1)$ (anyhow $Q_{2}$ packets will remain in system).

For all types of renovation policy the following condition on probabilies $q_{k}$, $q_{k}(\hat{q})(k \geqslant 1)$ and $q$ must be met:

$$
p_{k}+q_{k}=1, \quad p+\sum_{k=1}^{\infty} q(k)=1, \quad p^{(2)}+q=1,
$$

where $p_{k}(k \geqslant 1), p$ and $p^{(2)}$ are the probabilities that the served packet will leave the system without dropping any other packets from the buffer (if $\left.Q_{1}+1 \leqslant \hat{q}\right)$.

The minus of these policies is that the drop probability ceases to depend on the queue length and it is impossible to set the maximum drop probability. Models with three thresholds allow to get rid of this minus.

For the third model in [13] the steady-state probability distribution of packets $p_{i}(i \geqslant 0)$ in the system(for imbedded Markov chain), some probabilities were obtained and represented by geometric form (when the threshold $Q_{1}$ is overcomed):

$$
\begin{aligned}
p_{i} & =\sum_{j=i-1}^{Q_{1}} p_{j}(-\mu)^{j+1-i} \alpha^{(j+1-i)}(\mu)+ \\
& +p_{Q_{1}+1} g^{i-Q_{1}-2}\left(g-\alpha(\mu)-\int_{0}^{\infty} A(g, x) e^{-\mu x} d A(x)\right), \quad i=\overline{1, Q_{1}+1},
\end{aligned}
$$




$$
p_{i}=p_{Q_{1}+1} g^{i-\left(Q_{1}+1\right)}, \quad i>Q_{1}+1,
$$

where $g$ is the unique solution of the equation $g=\alpha(\mu(1-g Q(g)))$, and belongs to interval $(0 ; 1), \alpha(s)$ is the Laplase-Stieltjes transformation of interrarrival time distribution function $A(x), Q(g)$ is the probability generating function for probabilities $\pi(l, k)$ that $l$ packets will be served and $k$ packets will be dropped from the buffer,

$$
A(g, x)=\sum_{l=1}^{Q_{1}+1-i} \frac{(\mu x g)^{l}}{l !} \sum_{j=0}^{Q_{1}+1-i-l} \pi(l, j) g^{j} .
$$

Also the probability that the arriving packet will be dropped and sojourn time characteristics for dropped packets were obtained in form of integral equations.

\subsection{The mathematical model with renovation and tree thresholds}

In this part of the article models with renovation and three thresholds $\left(Q_{1}, Q_{2}\right.$ and $\left.Q_{3}\right)$ will be formulated. The first threshold $Q_{1}$ determines when packets start to be dropped, the second threshold $Q_{2}\left(Q_{2}>Q_{1}\right)$ sets the maximum value of drop probability, the third threshold $Q_{3}\left(Q_{3}<Q_{1}\right)$ determines the place to which packets may be dropped from the queue.

The following types of renovation mechanism may be defined. At the moment of the end of a packet service the current queue length $\hat{q}$ is compared with thresholds and if $\hat{q} \leqslant Q_{1}$ then no one of the packets from the buffer is dropped.

- If $Q_{1}+1 \leqslant \hat{q} \leqslant Q_{2}$ then the fixed size group of $k(k \geqslant 1)$ packets will be dropped with fixed probability $q_{k}$. If $\hat{q} \geqslant Q_{2}+1$ then the fixed size group of $k$ packets in the buffer will be dropped with maximal probability $q_{\max }\left(q_{k}<q_{\max } \leqslant 1\right.$, but $Q_{3}$ packeta anyhow will remain in the system.

- If $Q_{1}+1 \leqslant \hat{q} \leqslant Q_{2}$ then the fixed size group of $k$ packets will be dropped with probability $q_{k}(\hat{q}), 0<q_{k}(\hat{q})<q_{\max }$. If $\hat{q} \geqslant Q_{2}+1$ then the fixed size group of $k$ packets in the buffer will be dropped with maximal probability $q_{\max }$, but $Q_{3}$ packeta anyhow will remain in the system.

- If $Q_{1}+1 \leqslant \hat{q} \leqslant Q_{2}$ then the fixed size group of $k$ packets will be dropped with fixed probability $q_{k}$. If $\hat{q} \geqslant Q_{2}+1$ then all packets except the first $Q_{1}$ will be dropped with probability $q$.

- If $Q_{1}+1 \leqslant \hat{q} \leqslant Q_{2}$ then the fixed size group of $k$ packets will be dropped with probability $q_{k}(\hat{q})$. If $\hat{q} \geqslant Q_{2}+1$ then all packets except the first $Q_{1}$ will be dropped with probability $q$.

- If $Q_{1}+1 \leqslant \hat{q} \leqslant Q_{2}$ then the arbitrary size group of $k$ packets will be dropped with given probability $q(k)(0<q(k)<1)$. If $\hat{q} \geqslant Q_{2}+1$ then all packets except the first $Q_{1}$ will be dropped with probability $q$. 
For all types of renovation policy the following condition on probabilies $q_{1}$, $q_{k}, q(\hat{q}), q_{k}(\hat{q}), q(k), q_{\max }, q(k \geqslant 1)$ must be met:

$$
p_{k}+q_{k}=1, \quad p_{k}(\hat{q})+q_{k}(\hat{q})=1, \quad k \geqslant 1, \quad p+\sum_{k=1}^{\infty} q(k)=1,
$$

where $p_{k}, p_{k}(\hat{q})(k \geqslant 1)$ and $p$ are the probabilities that the served packet will leave the system without dropping any other packets from the buffer (if $\left.Q_{1}+1 \leqslant \hat{q} \leqslant Q_{2}\right)$, and $p_{\max }^{(2)}+q_{\max }=1, p^{(2)}+q=1, p_{\max }^{(2)}$ and $p^{(2)}$ are the probabilities that the served packet will leave the system without dropping any other packets from the buffer (if $\hat{q} \geqslant Q_{2}+1$ ).

\section{The comparison of some renovation models with RED algorithm}

In this section we will compare values of the probability of packet being dropped from the system $p^{\text {loss }}$ for RED and TailDrop algorithms [4], [6], [7] and values of the probability $p^{\text {loss }}$, obtained by formulas derived for queueing system with general renovation [11], queueing system with general renovation and feedback [33], [34] and queueing system with general renovation and two thresholds [12].

As can be see from the table 1 , according to the values of the $p^{\text {loss }}$, renovation mechanism can perform as good as RED in the wide range of the offered load $\rho$.

Table 1

The value of the loss probability for Taildrop, RED, general renovation, general renovation with feedback, general renovation with two thresholds

\begin{tabular}{|c|c|c|c|c|}
\hline \multicolumn{5}{|c|}{ Loss probability } \\
\hline \hline Taildrop & RED & renov. & ren-fd. & ren-thr. \\
\hline \multicolumn{5}{|c|}{$\rho=0.5$} \\
\hline 0 & 0.002 & 0.002 & 0.002 & 0.003 \\
\hline \multicolumn{5}{|c|}{$\rho=1$} \\
\hline 0.051 & 0.091 & 0.104 & 0.11 & 0.109 \\
\hline \multicolumn{5}{|c|}{$\rho=2$} \\
\hline 0.500 & 0.500 & 0.502 & 0.54 & 0.524 \\
\hline \multicolumn{5}{|c|}{$\rho=3$} \\
\hline 0.667 & 0.667 & 0.667 & 0.71 & 0.0 .679 \\
\hline
\end{tabular}




\section{Conclusion}

The main task of the authors in this work was to formulate different types of models with various renovation policies and one or more threshold values in a buffer of a system. These thresholds allows to control the drop probability of renovation mechanism. Some of the described models have already been researched or are in the process of research (links to scientific publications are provided)

The presented numerical experiments show that the results remain qualitatively the same for RED-type AQM with other dropping functions. Being defined by $N$ parameters, the renovation mechanism is very flexible and this constitutes its strength and weakness. By varying the values of the renovation probabilities $q(i)$, it is possible to carry out conditional optimisation, but good searching procedures are required here.

\section{Acknowledgments}

The publication has been funded by RFBR according to the research project No. 19-07-00739.

\section{References}

[1] F. Baker and G. Fairhurst. (Jul. 2015). "IETF Recommendations Regarding Active Queue Management. RFC 7567.," [Online]. Available: https://tools.ietf.org/html/rfc7567.

[2] M. Menth and S. Veith, "Active Queue Management Based on Congestion Policing (CP-AQM)," in. Jan. 2018, pp. 173-187. DOI: 10.1007/9783-319-74947-1_12.

[3] R. Adams, "Active Queue Management: A Survey," Communications Surveys \& Tutorials, IEEE, vol. 15, pp. 1425-1476, Jan. 2013. DOI: 10.1109/SURV.2012.082212.00018.

[4] A. Chydzinski and L. Chrost, "Analysis of AQM queues with queue size based packet dropping," Applied Mathematics and Computer Science, vol. 21, pp. 567-577, Sep. 2011. DOI: 10.2478/v10006-011-0045-7.

[5] A. Chydzinski and P. Mrozowski, "Queues with dropping functions and general arrival processes," PloS one, vol. 11, e0150702, Mar. 2016. DOI: 10.1371/journal. pone.0150702.

[6] M. Konovalov and R. Razumchik, "Numerical analysis of improved access restriction algorithms in a GI/G/1/N system," Journal of Communications Technology and Electronics, vol. 63, pp. 616-625, Jun. 2018. DOI: $10.1134 / \mathrm{S} 1064226918060141$.

[7] M. Konovalov and R. Razumchik, "Comparison of two active queue management schemes through the $\mathrm{M} / \mathrm{D} / 1 / \mathrm{N}$ queue," Informatika $i$ ee Primeneniya (Informatics and Applications), vol. 12, no. 4, pp. 9-15, 2018. DOI: $10.14357 / 19922264180402$. 
[8] S. Floyd and V. Jacobson, "Random Early Detection Gateways for Congestion Avoidance," IEEE/ACM Transactions on Networking, vol. 1, pp. 397-413, Sep. 1993. DOI: 10.1109/90.251892.

[9] K. Ramakrishnan, S. Floyd, and D. Black. (2001). "RFC3168: The Addition of Explicit Congestion Notification (ECN) to IP," [Online]. Available: https://tools.ietf .org/html/rfc3168.

[10] S. Floyd, R. Gummadi, and S. Shenker, "Adaptive RED: An Algorithm for Increasing the Robustness of RED's Active Queue Management," Sep. 2001.

[11] M. Konovalov and R. Razumchik. (2017). "Queueing systems with renovation vs. queues with RED. Supplementary Material." arXiv: 1709. 01477, [Online]. Available: https://arxiv.org/abs/1709.01477.

[12] I. Zaryadov and A. Korolkova, "The Application of model with general renovation to the analysis of characteristics of active queue management with random early detection (RED) [Primeneniye modeli s obobshchennym obnovleniyem $\mathrm{k}$ analizu kharakteristik sistem aktivnogo upravleniya ocheredyami tipa Random Early Detection (RED)]," T-Comm, no. 7, pp. 84-88, 2011, In Russian.

[13] H. Viana Carvalho Cravid et al., "The General Renovation as the Active Queue Management Mechanism. Some Aspects and Results," in Communications in Computer and Information Science. 2019, vol. 1141, pp. 488-502. DOI: $10.1007 / 978-3-030-36625-4 \_39$.

[14] V. Jacobson, K. Nichols, and K. Poduri, "RED in a Different Light," Tech. Rep., 1999.

[15] G. Fairhurst and M. Welzl. (2017). "The Benefits of Using Explicit Congestion Notification (ECN). RFC 8087," [Online]. Available: https: //tools.ietf.org/html/rfc3168.

[16] C. So-In, R. Jain, and J. Jiang, "Enhanced Forward Explicit Congestion Notification (E-FECN) scheme for datacenter Ethernet networks," in 2008 International Symposium on Performance Evaluation of Computer and Telecommunication Systems, 2008, pp. 542-546.

[17] C. Gomez, X. Wang, and A. Shami, "Intelligent active queue management using explicit congestion notification," in 2019 IEEE Global Communications Conference (GLOBECOM), Sep. 2019, pp. 1-6. DOI: 10.20944/preprints201909.0077.v1.

[18] S. Shahzad, E.-S. Jung, J. F. Chung M., and R. Kettimuthu, "Enhanced Explicit Congestion Notification (EECN) in TCP with P4 Programming," in 2020 International Conference on Green and Human Information Technology (ICGHIT), Feb. 2020. DOI: 10.1109/ICGHIT49656.2020. 00015.

[19] S. Wang, J. Zhang, T. Huang, T. Pan, J. Liu, and Y. Liu, "A-ECN Minimizing Queue Length for Datacenter Networks," IEEE Access, vol. 8, pp. 49 100-49 111, 2020. DOI: 10.1109/ACCESS. 2020. 2979216.

[20] K. Nichols and V. Jacobson, "Controlling queue delay," Communications of The $A C M-C A C M$, vol. 55, pp. 42-50, May 2012. DOI: 10.1145/ 2209249.2209264. 
[21] T. Hoeiland-Joergensen et al. (2018). "The Flow Queue CoDel Packet Scheduler and Active Queue Management Algorithm. RFC 8290," [Online]. Available: https://www.rfc-editor.org/info/rfc8290.

[22] S. Jung, J. Kim, and J.-H. Kim, "Intelligent active queue management for stabilized QoS guarantees in 5G mobile networks," IEEE Systems Journal, vol. PP, pp. 1-10, Aug. 2020. DOI: 10.1109/JSYST.2020.3014231.

[23] W.-C. Feng, D. Kandlur, D. Saha, and K. Shin, "BLUE: a new class of active queue management algorithms," University of Michigan, Tech. Rep., Sep. 2000. DOI: 10.1109/TNET . 2002.801399.

[24] W.-C. Feng, D. Kandlur, and D. Saha, "The BLUE active queue management algorithms," Networking, IEEE/ACM Transactions on, vol. 10, pp. 513-528, Sep. 2002. DOI: 10.1109/TNET. 2002.801399.

[25] C. Zhang, J. Yin, and Z. Cai, "RSFB: a Resilient Stochastic Fair Blue algorithm against spoofing DDoS attacks," in 2009 9th International Symposium on Communications and Information Technology, 2009, pp. 15661567. DOI: $10.1109 /$ ISCIT . 2009.5341033.

[26] G. Da-gang, "A New Adaptive BLUE Algorithm," in 2010 International Conference on Electrical and Control Engineering, 2010, pp. 2601-2605. DOI: 10.1109/iCECE. 2010.638.

[27] T. Hoiland-Jorgensen, D. Taht, and J. Morton, "Piece of CAKE: A Comprehensive Queue Management Solution for Home Gateways," in 2018 IEEE International Symposium on Local and Metropolitan Area Networks (LANMAN), Jun. 2018, pp. 37-42. DOI: 10.1109/LANMAN. 2018. 8475045.

[28] J. Palmei, S. Gupta, P. Imputato, J. Morton, M. Tahiliani, S. Avallone, and D. Taht, "Design and Evaluation of COBALT Queue Discipline," in 2019 IEEE International Symposium on Local and Metropolitan Area Networks (LANMAN), Jul. 2019, pp. 1-6. DOI: 10.1109/LANMAN. 2019. 8847054.

[29] W.-C. Feng, "Improving Internet Congestion Control and Queue Management Algorithms," Ph.D. dissertation, The University of Michigan, 1999.

[30] A. V. Korolkova, D. S. Kulyabov, and A. I. Chernoivanov, "On the classification of RED algorithms," Bulletin of Peoples' Friendship University of Russia. Series Mathematics. Information Sciences. Physics, no. 3, pp. 34-46, 2009, In Russian.

[31] A. Kreinin, "Queueing systems with renovation," Journal of Applied Mathematics and Stochastic Analysis, vol. 10, pp. 431-443, Jan. 1997. DOI: $10.1155 / \mathrm{S} 1048953397000464$.

[32] P. P. Bocharov and I. S. Zaryadov, "Probability Distribution in Queueing Systems with Renovation," Bulletin of Peoples' Friendship University of Russia. Series Mathematics. Information Sciences. Physics, pp. 15-25, 2007. 
[33] E. Bogdanova et al., "Characteristics of lost and served packets for retrial queueing system with general renovation and recurrent input flow," Communications in Computer and Information Science, vol. 919, pp. 327-340, 2018. DOI: 10.1007/978-3-319-99447-5_28.

[34] I. Zaryadov, E. Bogdanova, T. Milovanova, S. Matushenko, and D. Pyatkina, "Stationary Characteristics of the GI/M/1 Queue with General Renovation and Feedback," in 10th International Congress on Ultra Modern Telecommunications and Control Systems and Workshops (ICUMT), Nov. 2018, pp. 1-6. DOI: 10.1109/ICUMT . 2018.8631244.

[35] I. Zaryadov and A. Pechinkin, "Stationary time characteristics of the $G I / M / n / \infty$ system with some variants of the generalized renovation discipline," Automation and Remote Control, vol. 70, pp. 2085-2097, Dec. 2009. DOI: $10.1134 /$ S0005117909120157.

[36] I. Zaryadov, "Queueing systems with general renovation," in International Conference on Ultra Modern Telecommunications and Workshops, Oct. 2009, pp. 1-4. DOI: 10.1109/ICUMT. 2009.5345382.

[37] I. Zaryadov, R. Razumchik, and T. Milovanova, "Stationary waiting time distribution in $\mathrm{G}|\mathrm{M}| \mathrm{n} \mid \mathrm{r}$ with random renovation policy," in Communications in Computer and Information Science, vol. 678, Feb. 2016, pp. 349-360. DOI: 10.1007/978-3-319-51917-3_31.

[38] E. V. Bogdanova, T. A. Milovanova, and I. S. Zaryadov, "The analysis of queuing system with general service distribution and renovation," RUDN Journal of Mathematics, Information Sciences and Physics, vol. 25, no. 1, pp. 3-8, 2017. DOI: 10.22363/2312-9735-2017-25-1-3-8.

[39] I. S. Zaryadov, E. V. Bogdanova, and T. A. Milovanova, "Probabilitytime characteristics of $\mathrm{M}|\mathrm{G}| 1 \mid 1$ queueing system with renovation," in CEUR Workshop Proceedings, vol. 1995, 2017, pp. 125-131.

\section{For citation:}

H. Viana Carvalho Cravid, I. S. Zaryadov, T. A. Milovanova, Queueing systems with different types of renovation mechanism and thresholds as the mathematical models of active queue management mechanism, Discrete and Continuous Models and Applied Computational Science 28 (4) (2020) 305-318. DOI: $10.22363 / 2658-4670-2020-28-4-305-318$.

\section{Information about the authors:}

Viana Carvalho Cravid, Hilquias - post-graduate student of Department of Applied Probability and Informatics of Peoples' Friendship University of Russia (RUDN University) (e-mail: hilvianamat1@gmail .com, phone: +7(495)9550927, Scopus Author ID: 57212930802)

Zaryadov, Ivan S. — Candidate of Physical and Mathematical Sciences, assistant professor of Department of Applied Probability and Informatics of Peoples' Friendship University of Russia (RUDN University); Senior Researcher of Institute of Informatics Problems of Federal Research Center "Computer Science and Control" Russian Academy of Sciences (e-mail: zaryadov-is@rudn.ru, phone: +7(495)9550927, ORCID: https://orcid.org/0000-0002-7909-6396, ResearcherID: B-8154-2018, Scopus Author ID: 35294470000) 
Milovanova, Tatiana A. - Candidate of Physical and Mathematical Sciences, lecturer of Department of Applied Probability and Informatics of Peoples' Friendship University of Russia (RUDN University) (e-mail: milovanova-ta@rudn.ru, phone: +7(495)9550927, ORCID: https://orcid.org/0000-0002-9388-9499, Scopus Author ID: 26641495400) 
УДК 519.872:519.217

PACS 07.05.Tp, 02.60.Pn, 02.70.Bf

DOI: $10.22363 / 2658-4670-2020-28-4-305-318$

\title{
Системы массового обслуживания с различными видами обновления и порогами как математические модели алгоритмов активного управления очередями
}

\author{
Илкиаш Виана Карвалью Кравид ${ }^{1}$, Иван С. Зарядов ${ }^{1,2}$, \\ Татьяна А. Милованова ${ }^{1}$
}

1 Российский университет дружбъ народов

ул. Миклухо-Маклая, д. 6, Москва, 117198, Россия

2 Институт проблем информатики

Федеральный исследовательский иентр «Информатика и управление» РАН ул. Вавилова, д. 44, корп. 2, Москва, 119333, Россия

Работа посвящена некоторым аспектам использования механизма обновления (различные варианты обновления рассмотрены, определения и краткий обзор представлены) с одним или несколькими порогами в качестве математических моделей механизмов активного управления очередями.

Описаны системы массового обслуживания, в которых реализован механизм обновления с порогами, позволяющий управлять числом заявок в системе путем их сброса из накопителя в зависимости от значения некоторого управляющего параметра и пороговых значений. Сброс заявок из накопителя происходит в момент окончания обслуживания заявки на приборе, что отличает данный механизм сброса от RED-подобных алгоритмов, для которых сброс возможен в момент поступления в систему.

Представлены модели с одним, двумя или тремя порогами. В этих моделях пороговые значения определяют не только место, с которого в накопителе начинается сброс заявок, но и до какой позиции заявки могут быть сброшены. Для некоторых из описываемых моделей уже получены аналитические и численные результаты (ссылки на работы представлены), но большая часть моделей находится в процессе изучения, поэтому представлены только описания и некоторые текущие данные.

Приведены некоторые результаты сравнения классического алгоритма RED с механизмом обновления.

Ключевые слова: система массового обслуживания, активное управление очередью, обновление, обобщенное обновление, функция сброса, пороговый механизм, контроль перегрузок сети 